Artículo

\title{
Evolución de la especialización y competitividad de la producción de limón en México
}

\author{
Juan Manuel Vargas-Canales ${ }^{1 \S}$ \\ Diana Laura Guido-López ${ }^{1}$ \\ Benito Rodríguez-Haros ${ }^{1}$ \\ Tzatzil Isela Bustamante-Lara ${ }^{1}$ \\ Joaquín Huitzilihuitl Camacho-Vera² \\ Sergio Orozco-Cirilo ${ }^{1}$
}

${ }^{1}$ Departamento de Estudios Sociales-División de Ciencias Sociales y Administrativas-Campus CelayaSalvatierra-Universidad de Guanajuato. (dianalpz_96@hotmail.com; benus27@yahoo.com; ti.bustamantel@ugto.mx; orozcosergio@ugto.mx). ${ }^{2}$ División de Estudios de Posgrado-Universidad de la Sierra Sur. (camachovera@yahoo.com.mx).

${ }^{\S}$ Autor para correspondencia: jm.vargas@ugto.mx.

\section{Resumen}

El objetivo de esta investigación fue estudiar la especialización y competitividad de la producción de limón en México. Los métodos de análisis fueron el cociente de localización, el Shift and Share y se integró un análisis de especialización y competitividad. Los resultados muestran una reconfiguración en la estructura productiva. Destacan a nivel nacional nueve estados especializados y competitivos. Los estados con mayores perspectivas de crecimiento de esta actividad son los que integran la costa del Golfo de México. La especialización y competitividad se relaciona con las condiciones naturales de las regiones productoras, la demanda del mercado y el cambio tecnológico.

Palabras clave: comercio internacional, especialización inteligente, ventajas comparativas.

Recibido: junio de 2020

Aceptado: agosto de 2020 


\section{Introducción}

Los cítricos son el principal y más popular cultivo frutícola del mundo (Donkersley et al., 2018; Sharma et al., 2018), se cultivan en la mayoría de las regiones tropicales y subtropicales (Agustí et al., 2014). Su producción aumenta cada año debido al incremento de la demanda de los consumidores (Sharma et al., 2017). Su cultivo, no solo es importante en términos económicos, también por sus beneficios nutricionales en humanos y animales (Sharma et al., 2018), por lo que están ganando popularidad en la industria alimentaria de todo el mundo, Mustafa (2015). Durante las últimas décadas, su producción, consumo y transformación se han expandido enormemente de la mano de la intensificación del comercio internacional (Liu et al., 2012).

El principal producto de la industria de los cítricos es el jugo, utilizado en muchas bebidas o como ingrediente en muchos alimentos (Zema et al., 2018). Además, se obtienen otros productos como mermeladas, jaleas, cáscaras confitadas, aromatizantes y saborizantes para bebidas, aceites y esencias (Mustafa, 2015; Sharma et al., 2017, 2018; Zema et al., 2018). En general, los cítricos contienen cantidades considerables de lípidos, azúcares y ácidos.

También, carbohidratos insolubles, enzimas, flavonoides, limonina, isolimonina, aceite de cáscara, componentes volátiles, pigmentos, vitaminas y minerales (Sharma et al., 2017). Contienen compuestos bioactivos como antioxidantes, anticancerígenos, antitumorales y antiinflamatorios (Sharma et al., 2017). Lo que le confiere una de las características más deseables y demandadas en la actualidad por la sociedad.

Con respecto al comercio internacional, los principales exportadores son España ( $\$ 798 \mathrm{M})$, México $(\$ 508 \mathrm{M})$, Turquía $(\$ 336 \mathrm{M})$, Argentina $(\$ 327 \mathrm{M})$ y Sudáfrica $(\$ 312 \mathrm{M})$. Los principales importadores son Estados Unidos de América $(\$ 477 \mathrm{M})$, Alemania $(\$ 310 \mathrm{M})$, los Países Bajos $(\$ 246 \mathrm{M})$, Francia ( $\$ 239 \mathrm{M})$ y el Reino Unido $(\$ 181 \mathrm{M})$ (Observatory of Economic Complexity, 2018). En México, el limón es uno de los productos agrícolas más exportados (SIAP, 2017), siendo Estados Unidos de América el principal mercado (Donkersley et al., 2018).

En los últimos años, la citricultura mexicana ha experimentado modificaciones importantes, en la superficie de producción y en la localización de la producción (Hernández et al., 2017). A nivel nacional se producen tres especies de limón: mexicano, persa e italiano y destacan en volumen de producción los estados de Michoacán, Veracruz, Oaxaca, Colima y Tamaulipas (SIAP, 2017).

En ese sentido y derivado de la globalización, la estructura económica de los países se ha transformado a un ritmo mayor durante los últimos años. A las economías nacionales se han integrado actores económicos diversos, que cooperan y compiten a la vez en los mismos mercados, con lo cual llegan a constituirse sistemas económicos y productivos muy complejos (VargasCanales et al., 2016; Vargas-Canales et al., 2018). Además, la creciente integración comercial y financiera entre los distintos grupos de países permite observar cierta sincronización de la vida económica, que es promovida por el modelo de competitividad en el que se encuentran inmersos.

El término sincronización se refiere al movimiento en fase de los ciclos económicos de dos o más países (Mejía et al., 2006). Es conveniente aclarar que no sólo se trata de una sincronización económica o comercial, sino de patrones de vida. Por lo tanto, para adaptarse a esa dinámica, 
altamente competitiva, es necesario hacer coincidir, también, las actividades productivas y las innovaciones tecnológicas que mejor respondan a los ciclos económicos de reproducción de capital (Vargas-Canales et al., 2016). Es preciso referir que la adaptación es un proceso clave en el capitalismo y en general su desarrollo se corresponderá con el paradigma tecnológico del ciclo en cuestión (Jiménez, 2014).

De modo que, en la actualidad el comercio internacional tiene grandes efectos en prácticamente todos los aspectos económicos y ámbitos de la sociedad. Para autores como Martin (2018), es posible observar efectos positivos para el consumidor, ganancias de productividad de las empresas, cambios en los precios y transformación de los patrones de consumo. Además, la participación en los mercados internacionales permite aprovechar las diferencias regionales en cuanto a dotación de recursos, capacidades y habilidades.

Se argumenta también que el comercio internacional permite que los consumidores se beneficien al obtener una mayor variedad de productos y de mejor calidad. Más aún, en el largo plazo y derivado de las fuerzas del mercado, se dan transformaciones de las estructuras productivas y los cambios generan una tendencia hacia la especialización de las regiones.

En los últimos años México ha suscrito diversos acuerdos comerciales con varios países, dentro de los que destaca el Tratado de Libre Comercio con América del Norte (TLCAN), por los volúmenes comerciales involucrados y por la importancia relativa para las economías que participan en él. Dicho tratado implicó, entre otras cosas, en la eliminación de aranceles y de la mayoría de las barreras comerciales no arancelarias sobre una amplia gama de productos agrícolas, e incluyó disposiciones sobre normas de origen, salvaguardias y normas sanitarias y fitosanitarias (Ghazalian, 2017).

El impacto económico que ha tenido el TLCAN es difícil de medir dado que el comercio internacional está influenciado por numerosas variables (Villareal y Fergusson, 2017). Sin embargo, se han encontrado beneficios económicos y sociales para la economía mexicana en su conjunto, pero estos no se han distribuido uniformemente en todo el país (Beghin, 2015; Blecker y Esquivel, 2010; Lederman et al., 2005; Villareal y Fergusson, 2017).

La realidad es que a partir de su firma se han generado diversas transformaciones en la estructura productiva del país y distintos procesos de acoplamiento. Sin embargo, no todas las actividades productivas tienen vínculos importantes con el exterior. No obstante, esta sincronización parcial, sí es un mecanismo de choque que modifica la estructura productiva del país (Vargas-canales et al., 2016) y que estimula procesos de especialización productiva regional y local (Krugman, 1993).

Históricamente se han dado diferentes etapas de especialización agrícola. En ese sentido, la política mexicana ha buscado favorecer la concentración de las economías agropecuarias aprovechando la proximidad espacial y promoviendo el desarrollo de agroclústers y actividades colectivas (SAGARPA-FAO, 2013; Sánchez, 2014). El sector agrícola mexicano presenta una creciente especialización de los recursos productivos estratégicos regionales. Los cuales, se orientan a cultivos con un alto valor comercial en el mercado nacional e internacional. Dentro de los que destacan las hortalizas y frutas (González, 2013). 
Así, para lograr ventajas competitivas o ventajas comparativas sostenibles y sustentables en las regiones es importante contar con: 1) una dotación de recursos naturales únicos; 2) recursos humanos con amplios conocimientos relacionados con los sistemas productivos; 3) uso de tecnología agrícola apropiada; y 4) adecuadas interacciones del conjunto de agentes económicos. Sin embargo, lograr un circulo virtuoso de los elementos anteriores es casi imposible sin una política adecuada. En ese sentido, el enfoque de sistemas regionales de innovación, es una herramienta fundamental para el diseño y la implementación de estrategias de especialización inteligente (Asheim, 2018). La especialización inteligente se basa en la identificación de competencias centrales y potenciales de las regiones para hacer el proceso de innovación más eficiente (Vlčková et al., 2018).

De esta forma, sería posible promover regiones con la capacidad de mantener una adecuada dinámica es sus procesos innovativos, con identidad y sostenibilidad. En ese sentido, de acuerdo con Martin (2018), los desafíos más importantes se relacionan con la comprensión de las fuerzas motrices de los mercados agrícolas mundiales, en la política comercial y en garantizar la seguridad alimentaria. Esta política se sujeta a los cánones del libre comercio bajo la idea de aprovechar los productos baratos del exterior para favorecer a los consumidores locales y generar divisas a través de los productos de exportación.

La idea de que el mercado es la principal fuerza detrás de la especialización se remonta, al menos, a Adam Smith (Smith, 1976). Para Smith, un mercado más grande permite una mayor división del trabajo y mayor especialización (Emran y Shilpi, 2012). Lo que asegura una demanda adecuada de capacidades, habilidades y productos especializados. De manera simultanea, desde los años ochenta se han desarrollado esfuerzos impresionantes por explicar la dinámica económica regional.

De acuerdo con Capello (2017), el conocimiento acumulado durante décadas de la economía regional ha logrado incorporar la dimensión espacio en el análisis del funcionamiento del mercado. Sin embargo, aunque esta idea ha sido una parte integral del pensamiento económico a lo largo de los últimos siglos, es sorprendente que casi no existan análisis formales del papel del mercado en la determinación del patrón de especialización y competitividad regional (Emran y Shilpi, 2012).

Para el caso mexicano, el limón es uno de los productos más vinculado al comercio internacional, que presenta un gran dinamismo a nivel nacional e internacional. De acuerdo con lo anterior y considerando que la producción agrícola responde a la demanda del mercado, y que ésta se vio acentuada con la firma del TLCAN (Cruz-Delgado et al., 2013), es posible que, a partir de la entrada en vigor de este tratado, se inició una reconfiguración de la estructura productiva, dando como resultado, en México, un proceso de especialización productiva.

Dado lo anterior, el objetivo de esta investigación fue analizar el comportamiento de la producción de limón en México de 1980 a 2016, mediante técnicas de análisis regional, a fin de detectar los efectos del mercado relacionados con su especialización y competitividad. Lo anterior, es de utilidad práctica para los tomadores de decisiones, ya que es un insumo básico para el diseño de políticas agrícolas específicas. 


\section{Materiales y métodos}

En este estudio se consideraron a los 32 estados de la República Mexicana y sus municipios como las unidades geográficas fundamentales para analizar el comportamiento de la especialización y competitividad de las regiones productoras de limón. Para el estudio, se utilizó el valor de la producción del limón como variable de análisis dado el interés para definir las características de su peso relativo sobre las diferentes economías de las entidades.

La información en cuanto al valor de la producción se obtuvo una serie de datos con una periodicidad anual del Servicio de Información Agroalimentaria y Pesquera (SIAP, 2018). Con esta información se construyó una base de datos del valor de la producción agrícola del cultivo del limón para los años 1980-2016. Posteriormente, se determinó el cociente de localización y un análisis de tendencias estructurales a partir del índice Shift and Share. En estos análisis, se consideró al subsector pecuario y agrícola como un solo bloque excluyendo el producto que se plantea analizar, a fin de tener la comparación del cultivo de limón con respecto al total de la economía del sector agropecuario.

La información se organizó a manera de una matriz de doble entrada sector-región (SEC-REG), colocando al sector agrícola y pecuario en las columnas y las regiones en las filas (Boisier, 1980). Una vez construida la matriz SEC-REG, se procedió a realizar el cálculo del cociente de localización (CL) y el análisis Shift and Share.

Para estimar el cociente de localización se utilizó la siguiente ecuación: $C L_{i}=\frac{\frac{x_{i j}}{\sum_{\mathrm{i}} x_{i j}}}{\frac{\sum_{j} x_{i j}}{\sum_{i} \Sigma_{j} X_{i j}}}$ donde: $C L_{i}=$ cociente de localización; $X=$ variable de análisis; $i=$ corresponde al sector; y $\mathrm{j}=$ corresponde a la región (en este caso, cada entidad federativa y municipio). De acuerdo con este cociente, se puede afirmar que existe especialización relativa del sector i en la región $\mathrm{j}$ cuando su valor es mayor que 1 (Boisier, 1980; Arias y Fortich, 2010).

El coeficiente se interpreta como una medida de concentración geográfica, que ubica la ventaja de cada sector dentro de un grupo de regiones. Cuanto más se acerque el valor del CL a cero, habrá un menor grado de concentración del producto analizado en la región y viceversa. Lo anterior implica que cuanto más difiera la estructura económica de la región y la del país en su conjunto, mayor será su nivel de especialización (Mulligan y Schmidt, 2005; Gómez-Zaldívar et al., 2017).

Para el cálculo del análisis Shift and Share, se utilizó la siguiente ecuación (Boisier, 1980):

$\mathrm{X}_{\mathrm{ij}}^{\prime}-\mathrm{X}_{\mathrm{ij}}=\Delta \mathrm{X}_{\mathrm{ij}}=\mathrm{X}_{\mathrm{ij}} \mathrm{r}+\mathrm{X}_{\mathrm{ij}}\left(\mathrm{r}_{\mathrm{i}} \mathrm{r}\right)+\mathrm{X}_{\mathrm{ij}}\left(\mathrm{r}_{\mathrm{ij}}-\mathrm{r}\right) \mathrm{r}=\frac{\sum_{\mathrm{i}=1}^{\mathrm{S}} \sum_{\mathrm{j}=1}^{\mathrm{R}}\left(\mathrm{X}_{\mathrm{ij}}^{\prime} \mathrm{X}_{\mathrm{ij}}\right)}{\sum_{\mathrm{i}=1}^{\mathrm{S}} \sum_{\mathrm{j}=1}^{\mathrm{R}} \mathrm{X}_{\mathrm{ij}}} \mathrm{r}_{\mathrm{i}}=\frac{\sum_{\mathrm{j}=1}^{\mathrm{R}}\left(\mathrm{X}_{\mathrm{i}}^{\prime} \mathrm{X}_{\mathrm{ij}}\right)}{\sum_{\mathrm{j}=1}^{\mathrm{R}} \mathrm{X}_{\mathrm{ij}}} \mathrm{r}_{\mathrm{ij}}=\frac{\mathrm{X}_{\mathrm{ij}} \mathrm{X}_{\mathrm{ij}}}{\mathrm{X}_{\mathrm{ij}}}$. Donde: $\mathrm{X}_{\mathrm{ij}} \mathrm{r}=$ corresponde al efecto total; $X_{i j}\left(r_{i}-r\right)$ se trata del efecto sectorial o estructural; $X_{i j}\left(r_{i j}-r\right)$, concierne al efecto regional o competitivo. El análisis de cambio y participación evalúa el diferencial del crecimiento de los sectores analizados en las regiones estudiadas. Éste es uno de los métodos de análisis dinámico más usado, en virtud de sus posibilidades analíticas y de la información necesaria para construirlo (Dunn, 1960). 
Fue planteado en la década de los sesenta, con lo cual se trató de responder a preguntas tales como, ¿cuáles regiones muestran mayor crecimiento? y si este último se puede atribuir a: i) un efecto global o nacional (suma de regiones); ii) un efecto sectorial (estructural); y iii) un efecto regional (competitivo) (Camacho Vera et al., 2017).

En cuanto al efecto sectorial, éste expresa el impacto positivo o negativo del crecimiento de un sector específico, por arriba o por debajo de la tasa de crecimiento nacional. El efecto regional o competitivo recoge el dinamismo de un sector en una región, contrastándolo con ese mismo sector en el ámbito nacional (Boisier, 1980; Camacho et al., 2017). Finalmente, se realizó una integración de los indicadores para su mejor comprensión y para obtener una prospectiva del comportamiento de la especialización y competitividad de la producción de limón.

Así, para el análisis Shift and Share, si un estado tiene un efecto positivo en los tres ámbitos (nacional, sectorial y regional), obtendrá un valor máximo de 3 y si tienen efecto negativo tiene 0 y para el caso de CL, el efecto positivo o negativo de determinó en función de su cambio en el periodo de análisis (Cuadro 1).

\section{Cuadro 1. Método de integración de los indicadores para determinar especialización y competitividad.}

\begin{tabular}{|c|c|c|c|c|c|}
\hline $\begin{array}{c}\text { Efecto } \\
\text { nacional }\end{array}$ & $\begin{array}{c}\text { Efecto } \\
\text { sectorial }\end{array}$ & $\begin{array}{l}\text { Efecto } \\
\text { regional }\end{array}$ & $\begin{array}{c}\Delta \mathrm{CL} \\
(1981-2016)\end{array}$ & \multirow{2}{*}{$\begin{array}{l}\text { Especialización y } \\
\text { competitividad }\end{array}$} & \multirow[t]{2}{*}{ Valor } \\
\hline \multicolumn{3}{|c|}{ Shift and Share } & Especialización & & \\
\hline- & - & - & - & - & 0 \\
\hline+ & - & - & - & + & 1 \\
\hline+ & + & - & - & ++ & 2 \\
\hline+ & + & + & - & +++ & 3 \\
\hline+ & + & + & + & ++++ & 4 \\
\hline
\end{tabular}

Elaboración con base en Boisier (1980); Mulligan y Schmidt (2005); Arias y Fortich (2010); Camacho et al. (2017); Gómez-Zaldívar et al. (2017).

\section{Resultados y discusión}

\section{Cociente de localización}

En los últimos años se ha incrementado la producción y comercialización de limón en México. Los resultados del cociente de localización muestran una fuerte expansión de especialización regional de la producción de limón. Como puede observarse para 1980, en varios estados de la república el cultivo de limón tenía una importancia relativa considerable con respecto al total de su economía agropecuaria (Figura 1).

En seis estados de la república la producción de limón superaba el comportamiento nacional, en el caso de Colima hasta 40 veces más. Para 2016 es posible observar un cambio importante en cuanto a la especialización en este cultivo, se reduce el peso específico del estado de Colima, Michoacán y Guerrero, aumenta el de Veracruz, Yucatán y Tabasco. Por otra parte, aparecen como especializados Oaxaca, Quintana Roo y Tamaulipas (Figura 2). 


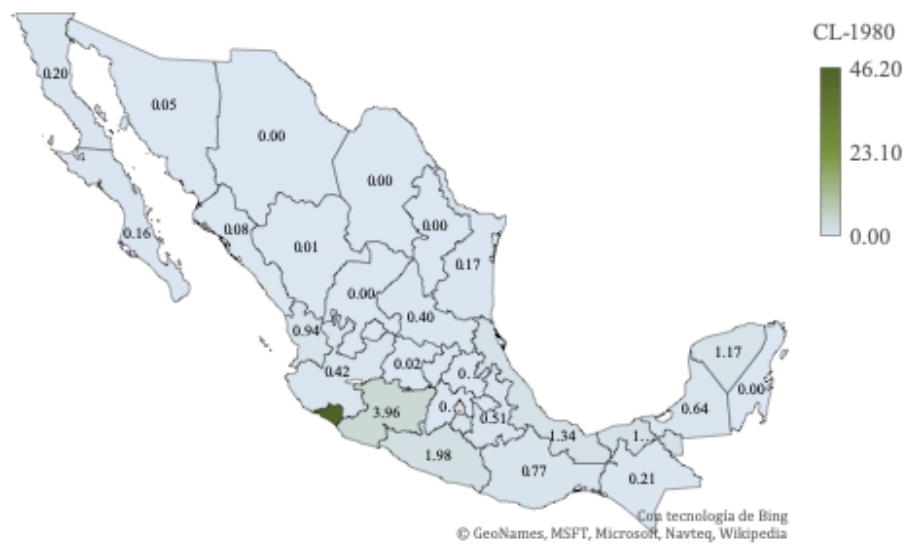

Figura 1. Especialización de la producción de limón en México en 1980.

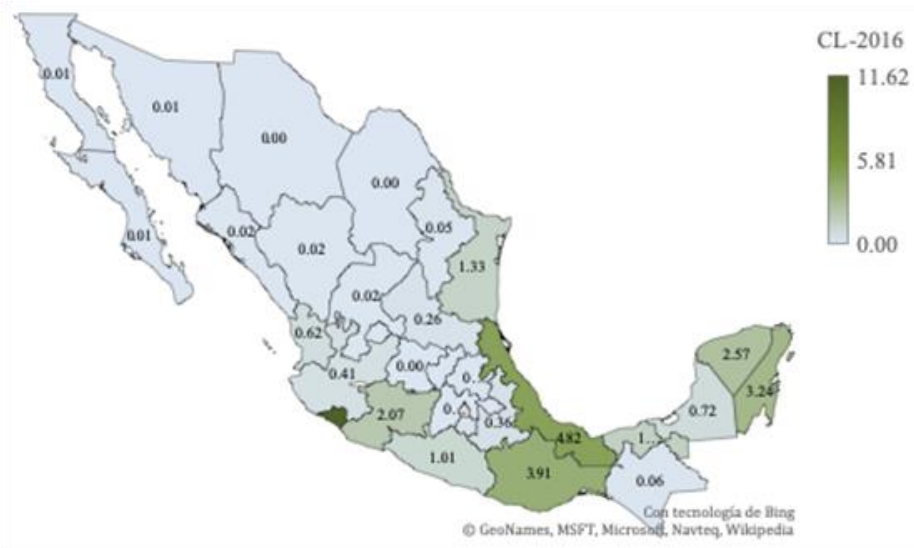

Figura 2. Especialización de la producción de limón en México en 2016.

Con respecto a la especialización a nivel local, en el Cuadro 2 se presentan los siete estados y sus municipios con el mayor nivel de especialización en la república mexicana. Es posible observar que la especialización de la producción de limón presenta una distribución espacial relativamente equilibrada. Es decir, la importancia relativa con respecto al total de su economía agropecuaria en los municipios especializados es similar, con excepción de Manzanillo en Colima; Santiago Laollaga en Oaxaca y González en Tamaulipas (Cuadro 2).

Cuadro 2. Especialización local de la producción de limón en México (2016).

\begin{tabular}{ccc}
\hline Estado & Municipio & CL \\
\hline Colima & Manzanillo & 19.03 \\
Veracruz & Martínez de La Torre & 1.01 \\
& Tlapacoyan & 1.01 \\
& Atzalan & 1.01 \\
& Coxquihui & 1.01 \\
& San Rafael & 1.01 \\
& Carrillo Puerto & 1.01 \\
& Misantla & 1.01 \\
\hline
\end{tabular}




\begin{tabular}{ccc}
\hline Estado & Municipio & CL \\
\hline & Nautla & 1.01 \\
& Paso del Macho & 1.01 \\
Oaxaca & Papantla & 1.01 \\
Quintana Roo & Santiago Laollaga & 37.93 \\
Yucatán & José María Morelos & 1.01 \\
& Temozón & 1.27 \\
& Oxkutzcab & 1.04 \\
& Peto & 1.04 \\
& Dzán & 1.03 \\
& Ticul & 1.03 \\
& Maní & 1.03 \\
& Tzucacab & 1.03 \\
Tabasco & Teabo & 1.02 \\
Tamaulipas & Huimanguillo & 1.1 \\
& González & 11.52 \\
& Gómez Farías & 1.01 \\
& Padilla & 1.01 \\
\hline
\end{tabular}

\section{Análisis Shift and Share}

Los resultados del análisis Shift and Share indican que nueve estados presentaron ventajas competitivas en la producción del cultivo del limón con respecto a otras regiones productoras de México. Los nueve estados presentan efecto positivo a nivel global (suma de regiones), sectorial (estructural) y regional (competitivo) (Figura 3). La producción de limón de estas regiones, en el periodo de análisis, tuvo un mejor comportamiento con respecto al crecimiento agrícola nacional y al crecimiento del sector. En decir, se presentaron las mejores condiciones para la expansión y el comercio del cultivo.

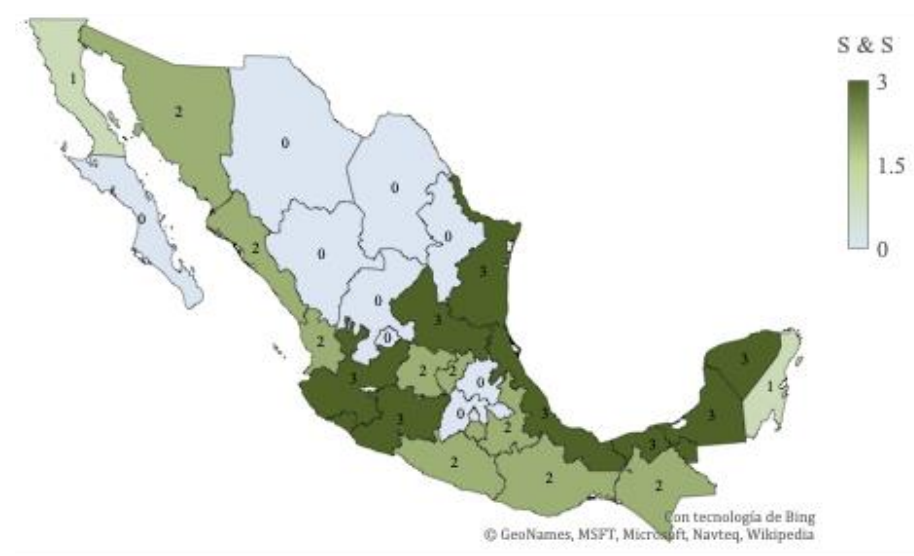

Figura 3. Análisis Shift and Share de la producción de limón en México (1980-2016). 


\section{Especialización y competitividad}

Con respecto al análisis integrador sobre la especialización y competitividad, los resultados muestran que los estados que integran la Costa del Golfo de México son los que tiene valores más altos (Figura 4). Lo anterior, debido a sus características naturales y la evolución de sus sistemas de producción de limón, lo que sugiere, será la región más especializada y competitiva. En segundo nivel se encuentra Colima, Michoacán, Jalisco, San Luis Potosí y Oaxaca. Lo que indica que estos estados también presentan condiciones para el desarrollo de esta actividad. Otro aspecto que sobresale es que el cultivo se ha posicionado como una buena alternativa y ha aumentado su importancia en la economía.

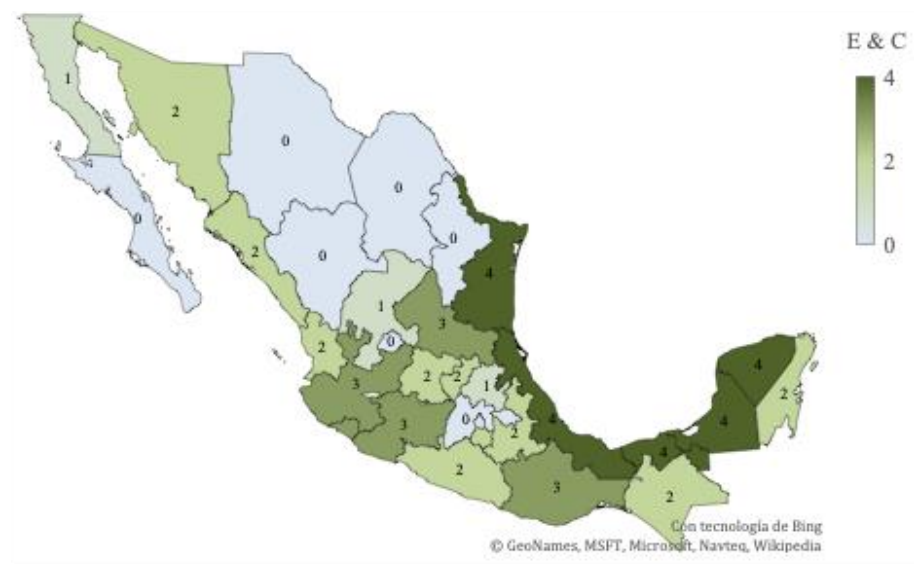

Figura 4. Prospectiva sobre la especialización y competitividad de la producción de limón en México.

El cultivo de limón es uno de los principales frutales que se producen en México (Magaña et al., 2010; Ramírez et al., 2008). El aumento de su producción se relaciona con el éxito del cultivo en el mercado internacional (Liu et al., 2012). De esta forma, como un primer efecto de la fuerte demanda internacional la frontera de producción de México se expandió. Lo anterior, ocasionó cambios en la estructura productiva y en el grado de especialización en las regiones dónde se cultiva.

Es conveniente mencionar que, en cuanto a la producción de limas ácidas, la del limón mexicano (Citrus aurantifolia Swingle) es la de mayor importancia para el mercado nacional (Zea-Hernández et al., 2016) y su producción se concentra en Colima, Michoacán y Guerrero (Ramírez Abarca et al., 2008). En segundo lugar, se encuentra el limón persa (Citrus latifolia Tanaka), de expansión más reciente y se orienta a cubrir los mercados internacionales.

Su producción se concentra en Veracruz, Quintana Roo, Oaxaca, Yucatán y Tabasco (HernándezTrujillo y Botello-Triana, 2017). Por último, el limón amarillo o italiano (Citrus limon, Burm), cuya producción se orienta a satisfacer la demanda de la industria refresquera, se concentra en Tamaulipas y San Luis Potosí.

Es claro que el grado y la forma en que se reestructuró la producción se relaciona directamente con las condiciones naturales de las regiones en donde se localiza el cultivo, la demanda del mercado nacional e internacional, la infraestructura disponible y el cambio tecnológico de los sistemas de producción. De acuerdo con los resultados y a la evolución de sus sistemas de producción de limón, 
la región más especializada y competitiva será la que integran el Golfo de México en la producción de limón persa. De manera general se trata de regiones tropicales y subtropicales ideales para el cultivo (Agustí et al., 2014).

Lo anterior, se relaciona con dos factores determinantes: 1) Veracruz ha desarrollado una infraestructura importante relacionada con la producción de limón persa (Fernández-Lambert et al., 2015); 2) la cercanía relativa a EE. UU, que es el principal mercado a nivel mundial de limón persa, del cual México el principal proveedor (Arias y Suarez, 2016). Además, es importante mencionar que Veracruz lidera la producción de limón persa de exportación en la región y el resto de los estados productores de limón persa tiene un fuerte vínculo con su agroindustria.

Lo que no implica que el resto de las regiones productoras de limón desaparezca, pero es posible que no mantenga su carácter especializado. Sin embargo, como lo menciona Buendía (2013), la competitividad no surge de manera espontánea, sino que es el resultado de políticas apropiadas. La producción citrícola es una actividad que se realiza con propósitos de largo plazo (HernándezTrujillo y Botello-Triana, 2017), en este sentido, las políticas de fomento sobre infraestructura, investigación y desarrollo tecnológico debe construirse con la misma proyección en el tiempo.

Por otra parte, si bien es cierto que desde el punto de vista económico el modelo productivo basado en la especialización ha traído grandes beneficios, también, es de esperarse que tenga efectos negativos sobre los recursos naturales, perdida de diversidad, contaminación e incluso en la salud humana (Tolón y Lastra, 2010; Landeros-Sánchez et al., 2011). Es importante mencionar que la diversidad de especies estimula la productividad, la estabilidad y la resiliencia en ecosistemas (Hajjar et al., 2008; Cabell y Oelofse, 2012; Cadotte et al., 2012; Hooper et al., 2012).

Además, desde hace décadas se han cuestionado los efectos negativos de la dinámica de producción agroexportadora sobre los sistemas tradicionales de producción. Su cuestionamiento se relaciona incluso con la perdida de costumbres, tradiciones y saberes (Hernández Xolocotzi, 1988). También se argumenta que la dependencia excesiva de los flujos comerciales externos genera dependencia económica, se debilita la soberanía y se pone en riesgo a los sistemas alimentarios (Otero et al., 2013; Rubio, 2014; Ortíz et al., 2016). Asimismo, la agricultura intensiva y de monocultivo es altamente dependiente del uso de insumos externos como como fertilizantes, agroquímicos, combustibles, maquinaria, entre otros.

Derivado de lo anterior y considerando que los principales retos que tienen que enfrentar la agricultura mundial, los gobiernos y la sociedad en su conjunto, son los de satisfacer la demanda de alimentos y mantener niveles sustentables de los recursos naturales (Pérez y Landeros, 2009), es necesario cuestionarnos sobre la sustentabilidad de estos sistemas de producción. En ese mismo sentido, para que esta actividad permanezca como una alternativa viable para impulsar el desarrollo rural, los retos en materia de competitividad y productividad se deben abordar considerando la situación técnica, social, organizacional, económica y ambiental (Partida y Meza, 2017).

Por otra parte, en la actualidad no se ha puesto énfasis en la articulación y relación de lo anterior, ni entre los actores presentes en el territorio, que son quienes configuran los sistemas de producción. Además, no se han considerado las ventajas relacionadas con la proximidad espacial de las empresas en la propuesta de alternativas, como podría ser el caso de una integración tipo agroclúster. 
Estas limitantes dificultan el aprovechamiento pleno de las ventajas relacionadas con las economías de escala y los nichos de mercado potenciales (García-Sánchez et al., 2018). De acuerdo con Vargas-Canales et al. (2018), es necesario pensar en esquemas de desarrollo colectivo, que consideren las características e interacciones que se desarrollan y evolucionan en los territorios, a fin de proponer una política integral.

De tal forma que, el enfoque de sistemas regionales de innovación es una herramienta ideal y fundamental para el diseño y la implementación de estrategias de especialización inteligente (Asheim, 2018) de largo plazo, acordes con las particularidades de cada región y basadas en el conocimiento, la tecnología y el medio ambiente para lograr sistemas agroalimentarios sustentables.

\section{Conclusiones}

La producción de limón presenta cambios en su estructura productiva y en su especialización espacial. Dicha transformación se relaciona directamente con las condiciones naturales de las regiones que presentan especialización y competitividad, que son ideales para el desarrollo óptimo del cultivo. Pero sobre todo con la creciente demanda nacional e internacional que ha favorecido el desarrollo de infraestructura y al desarrollo de innovaciones tecnológicas que se han adoptado y adaptado de forma eficaz en los sistemas de producción.

La concentración espacial de la producción de limón se debe a las particularidades que les confieren ventajas comparativas con respecto a otros espacios geográficos. De manera general la especialización y competitividad, considerando el comportamiento histórico de este cultivo, se espera que se expanda aún más. En específico, se espera que el crecimiento más fuerte ocurra en los estados que integran la costa del Golfo de México y sea de limón persa.

Por otra parte, para que esta actividad permanezca como una alternativa viable para impulsar el desarrollo económico sostenido, los retos en materia de especialización y productividad se deben abordar desde una perspectiva sistémica y considerando aspectos técnicos, económicos, sociales y ambientales. En ese sentido, el enfoque de sistemas regionales de innovación es la herramienta ideal para implementar estrategias de especialización inteligente y lograr sistemas agroalimentarios sustentables.

\section{Literatura citada}

Agustí, M.; Mesejo, C.; Reig, C. and Martínez-Fuentes, A. 2014. Citrus production. In: Dixon, G. R. y Aldous, D. E. (Eds.). Horticulture: plants for people and paces. Vol. 1. Springer. Production Horticulture. 159-196 pp.

Arias, F. y Suarez, E. 2016. Comportamiento de las exportaciones de limón persa (Citrus latifolia tanaka) al mercado de los Estados Unidos. J. Agric. Animal Sci. 5(2):20-31.

Arias, V. J. A. y Fortich, P. F. J. 2010. El panorama teórico de la economía regional y los modelos de análisis territorial. Finanzas y Política Económica. 2(2):9-26.

Asheim, B. T. 2018. Smart specialisation, innovation policy and regional innovation systems: what about new path development in less innovative regions? Innovation. Eur. J. Soc. Sci. Res. 0(0):1-18. https://doi.org/10.1080/13511610.2018.1491001. 
Beghin, J. C. 2015. NAFTA: implications for Mexican and midwestern agriculture. Iowa Ag Review Online. 7(1):9-12.

Blecker, R. A. and Esquivel, G. 2010. NAFTA, trade and development. CESifo Forum. 4:17-30.

Boisier, S. 1980. Técnicas de análisis regional con información limitada. Cuaderno ILPES, Serie 2(27):1-184.

Buendía-Rice, E. A. 2013. El papel de la ventaja competitiva en el desarrollo económico de los países. Análisis Económico. 28(69):55-78.

Cabell, J. F. y Oelofse, M. 2012. An indicator framework for assessing agroecosystem resilience. Ecol. Soc. 17(1):1-18. https://doi.org/10.5751/ES-04666-170118.

Cadotte, M.; Dinnage, R. and Tilman, D. 2012. Phylogenetic diversity promotes ecosytem stability. Ecology. 93(8):S223-S233. https://doi.org/10.1890/11-0426.1.

Camacho-Vera, J.; Cervantes-Escoto, F.; Palacios-Rangel, M.; Cesín-Vargas, A. y OcampoLedesma, G. 2017. Especialización de los sistemas productivos lecheros en México: la difusión del modelo tecnológico Holstein. Rev. Mex. Cienc. Pec. 8(3):259-268. https://doi.org/10.22319/rmcp.v8i3.4191.

Capello, R. 2017. Seminal studies in regional and urban economics. Contributions from an Impressive Mind. Springer International Publishing. Cham, Switzerland. 455 p. https://doi.org/10.1007/978-3-319-57807-1.

Cruz-Delgado, D.; Leos-Rodríguez, J. A. y Altamirano-Cárdenas, J. R. 2013. México: factores explicativos de la producción de frutas y hortalizas ante la apertura comercial. Rev. Chapingo Ser. Hortic. 19(3):267-278. https://doi.org/10.5154/r.rchsh.2012.05.029.

Donkersley, P.; Silva, F. W. S.; Carvalho, C. M.; Al-Sadi, A. M. and Elliot, S. L. 2018. Biological, environmental and socioeconomic threats to citrus lime production. J. Plant Dis. Protec. 125(4):339-356. https://doi.org/10.1007/s41348-018-0160-x.

Dunn, E. 1960. A statistical and analytical technique for regional analysis. Papers in Reg. Sci. 6(1):97-112.

Emran, M. S. y Shilpi, F. 2012. The extent of the market and stages of agricultural specialization. Canad. J. Econ. 45(3):1125-1153. https://doi.org/10.1111/j.1540-5982.2012.01729.x.

Fernández-Lambert, G.; Aguilar-Lasserre, A. A.; Martínez-Castellanos, G. J.; Ruvalcaba-Sánchez, M. L. G.; Correa-Medina, J. G. y Martínez-Flores, J. L. 2015. Contexto y caracterización de la cadena de suministro del limón persa (Citrus latifolia Tanaka) en Veracruz-México. Conciencia Tecnológica. 50:21-31.

García-Sánchez, E. I.; Vargas-Canales, J. M.; Palacios-Rangel, M. I. y Aguilar-Ávila, J. 2018. Sistema de innovación como marco analítico de la agricultura protegida en la región centro de México. Cuadernos de Desarrollo Rural. 15(81):1-24. https://doi.org/10.11144/ Javeriana.cdr15-8.sima.

Ghazalian, P. L. 2017. The effects of NAFTA/CUSFTA on agricultural trade flows: an empirical investigation. Canad. J. Agric. Econ. 65(2):219-248. https://doi.org/10.1111/cjag. 12119.

Gómez-Zaldívar, M.; Mosqueda, M. T. y Duran, A. J. 2017. Localization of manufacturing industries and specialization in Mexican states: 1993-2013. Regional science policy y practice. 9(4):301-315. https://doi.org/10.1111/rsp3.12111.

González, H. 2013. Especialización productiva y vulnerabilidad agroalimentaria en México. Comercio Exterior. 63(2):21-36.

Hajjar, R.; Jarvis, D. I. and Gemmill-Herren, B. 2008. The utility of crop genetic diversity in maintaining ecosystem services. Agric. Ecosys. Environ. 123(4):261-270. https://doi.org/10.1016/j.agee.2007.08.003. 
Hernández-Trujillo, J. M. y Botello-Triana, J. 2017. El papel del entorno en las modificaciones de la estructura regional de la producción de limón y de naranja en México. Análisis Económico. 32(80):93-118.

Hernández-Xolocotzi, E. 1988. La agricultura tradicional en México. Comercio Exterior. 38(8):673-678.

Hooper, D. U.; Adair, E. C.; Cardinale, B. J.; Byrnes, J. E. K.; Hungate, B. A.; Matulich, K. L. and Connor, M. I. 2012. A global synthesis reveals biodiversity loss as a major driver of ecosystem change. Nature. 486(7401):105-108. https://doi.org/10.1038/nature11118.

Jimenez, B. Y. 2014. The long economic cycles and their dialectics with the capitalist development. Economía y Desarrollo. 151(1):44-55.

Krugman, P. 1993. Lessons of Massachussets for EMU. In: Gavazzi, F. and Torres, F. (Eds.). The transition to economic and monetary Union in Europe. New York: Cambridge University Press. 241-261 pp.

Landeros-Sánchez, C.; Moreno-Seceña, J.; Nikolskii Gavrilov, L. y Bakhlaeva Egorova, O. 2011. La biodiversidad en Veracruz: estudio de Estado. In: Cruz-Angón A. (Ed.). Impacto de la agricultura sobre la biodiversidad. Comisión Nacional para el Conocimiento de la Biodiversidad (CONABIO)- Gobierno del Estado de Veracruz, Universidad Veracruzana, Instituto de Ecología. 477-491 pp.

Lederman, D.; Maloney, W. F. and Serven, L. 2005. Lessons from NAFTA for Latin America and the Caribbean. Stanford University Press and the World Bank, Latin American Development Forum Series: Palo Alto, California and Washington, DC. 376 p.

Liu, Y.; Heying, E. and Tanumihardjo, S. A. 2012. History, global distribution, and nutritional importance of citrus fruits. Comprehensive reviews in food science and food safety, 11(6):530-545. https://doi.org/10.1111/j.1541-4337.2012.00201.x.

Magaña-Sánchez, A. P.; Padilla-Bernal, L. E. y Vargas-Hernández, J. G. 2010. Competitividad de las agroindustrias del limón pertenecientes al clúster de limón mexicano en Colima, México. Economía y Sociedad. 14(25):139-152.

Martin, W. 2018. A research agenda for international agricultural trade. Appl. Econ. Perspectives Policy. 40(1):155-173. https://doi.org/10.1093/aepp/ppx063.

Mejía, R. P.; Gutiérrez, A. E. E. y Farías, S. C. A. 2006. La sincronización de los ciclos económicos de México y Estados Unidos. Investigación Económica. 65(258):15-45.

Mulligan, G. F. and Schmidt, C. 2005. A note on localization and specialization. Growth and Change. 36(4):565-576. https://doi.org/10.1111/j.1468-2257.2005.00295.x.

Mustafa, N. 2015. Citrus essential oils: current and prospective uses in the food industry. Recent Patents Food, Nutr. Agric. 7(2):115-127. https://doi.org/10.2174/22127984076661 50831144239.

OEC. 2018. Lemons and limes, fresh or dried. Product Trade, Exporters and Importers. https://oec.world/en/profile/hs92/lemons-and-limes-fresh-or-dried.

Ortíz-Caldera, H.; Montes-Torres, M. D. y Jiménez-González, A. 2016. La reconversión productiva ¿desarrollo o retroceso? Educateconciencia. 10(11):13-25.

Otero, G.; Pechlaner, G. and Gürcan, E. C. 2013. The political economy of 'food security' and trade: uneven and combined dependency. Rural Sociol. 78(3):263-289. https://doi.org/10.1111/ruso.12011.

Partida-Zamora, M. y Meza-Ramos, E. 2017. La competitividad y la productividad del limón persa en Nayarit (México). Cuadernos Claeh. 36(105):127-140.

Pérez-Vázquez, A. y Landeros-Sánchez, C. 2009. Agricultura y deterioro ambiental. Elementos. 73(16)19-25. 
Ramírez-Abarca, O.; González-Razo, F. J.; Omaña-Silvestre, J.; Matus-Gardea, J.; Kido-Cruz, A.; Rebollar-Rebollar, S. y Ortíz-Rosales, M. 2008. Aspectos económicos de la producción de limón mexicano en los estados de Colima y Michoacán, México. Inceptum. 3(5):67-80.

Rubio-Vega, B. A. 2014. El dominio del hambre: Crisis de hegemonia y alimentos. Ed. Juan Pablos. México, DF. 270 p.

SAGARPA-FAO. 2013. Aglomeraciones productivas 'clusters': una vía para impulsar la competitividad del sector agroalimentario en México.

Sánchez-Cano, J. E. 2014. La política agrícola en México, impactos y retos. Rev. Mex. Agroneg. 18(35):946-956.

Sharma, K.; Mahato, N.; Cho, M. H. and Lee, Y. R. 2017. Converting citrus wastes into valueadded products: Economic and environmently friendly approaches. Nutrition. 34:29-46. https://doi.org/10.1016/j.nut.2016.09.006.

Sharma, K.; Mahato, N. and Lee, Y. R. 2018. Extraction, characterization and biological activity of citrus flavonoids. Reviews in Chem. Eng. 35(2):265-284. https://doi.org/10.1515/revce2017-0027.

SIAP. 2017. Servicio de Información Agroalimentaria y Pesquera. Atlas Agroalimentarios. Primera (Ed). México, DF.

SIAP. 2018. Servicio de Información Agroalimentaria y Pesquera. Datos abiertos.

Smith, A. 1976. An inquiry into the nature and causes of the wealth of nations. Chicago: University of Chicago Press. 563 p.

Tolón-Becerra, A. y Lastra-Bravo, X. 2010. La agricultura intensiva del poniente almeriense. Diagnóstico e instrumentos de gestión ambiental. Rev. Elec. Medioamb. -M+A. 8:18-40.

Vargas-Canales, J. M.; Palacios-Rangel, M. I.; Aguilar-Ávila, J. y Ocampo-Ledesma, J. G. 2016. Cambio tecnológico e innovación en agricultura protegida en Hidalgo, México. Tesis de Doctorado en Problemas Económico Agroindustriales. Universidad Autónoma Chapingo. Chapingo, Edo. México. 181 p.

Vargas-Canales, J. M.; Palacios-Rangel, M. I.; Aguilar-Ávila, J.; Ocampo-Ledesma, J. G.; Kreimer, P. and Ortiz-Martínez, G. 2018. Technological innovation in a case of protected agriculture in Mexico. Rev. Geog. Agríc. 61(2):9-38. https://doi.org/10.5154/ r.rga.2017.61.02.

Vargas-Canales, J. M.; Palacios-Rangel, M. I.; Aguilar-Ávila, J.; Ocampo-Ledesma, J. G. and Medina-Cuellar, S. E. 2018. Efficiency of small enterprises of protected agriculture in the adoption of innovations in Mexico. Estudios Gerenciales 34(146):52-62. https://doi.org/10.18046/j.estger.2018.146.2811.

Villareal, M. and Fergusson, I. F. 2017. The North American Free Trade Agreement (NAFTA). Congressional Research Services Report R42965. Washington, DC. 38 p. https://doi.org/ 10.1080/13563460802673366.

Vlčková, J.; Kaspř́iková, N. and Vlčková, M. 2018. Technological relatedness, knowledge space and smart specialisation: The case of Germany. Moravian Geographical Reports. 26(2):95108. https://doi.org/10.2478/mgr-2018-0008.

Zea-Hernández, L. O.; Saucedo-Veloz, C.; Cruz-Huerta, N.; Ramírez-Guzmán, M. E. and RoblesGonzález, M. M. 2016. Evaluation of post-harvest applications of gibberellic acid on the quality and shelf life of three varieties of Mexican lime. Rev. Chapingo Ser. Hortic. 22(1):17-26. https://doi.org/10.5154/r.rchsh.2015.01.005.

Zema, D. A.; Calabrò, P. S.; Folino, A.; Tamburino, V.; Zappia, G. and Zimbone, S. M. 2018. Valorisation of citrus processing waste: a review. Waste Management. 80:252-273. https://doi.org/10.1016/j.wasman.2018.09.024. 